\title{
Neurocisticercose em tronco cerebral: relato de caso e revisão da literatura
}

\author{
Luiz Eduardo Ribeiro Wanderley Filho ${ }^{1}$, Lucas Chaves Lelis ${ }^{1}$, Caio Sander Junior ${ }^{1}$, \\ Everton Barbosa², Wilson Faglione Junior², Carlos Antônio Guimarães Bastos ${ }^{3}$ \\ Serviço de Neurocirurgia do Hospital São Rafael, Salvador, BA, Brasil.
}

\section{RESUMO}

Descrever patologia rara em que há poucos relatos sobre a incidência em tronco cerebral (em torno de $8 \%$ dos casos), cujo tratamento adequado resulta em prognóstico favorável. Descrição de tratamento cirúrgico de paciente com neurocisticercose em tronco cerebral. Paciente evoluiu com regressão completa dos sintomas prévios após cirurgia. Na neurocisticercose, a definição do tratamento clínico ou cirúrgico está diretamente relacionada à localização, ao tamanho e ao número de lesões, diminuindo, assim, a mortalidade e a morbidade nessa patologia.

\section{PALAVRAS-CHAVE}

Neurocisticercose/etiologia, neurocisticercose/fisiopatologia, neurocisticercose/cirurgia, tronco encefálico.

\section{ABSTRACT}

Brainstem cysticercosis: case report and literature review

To describe a rare pathology, where there are few reports on the incidence of brain stem (about $8 \%$ of cases), whose proper treatment results in favorable prognosis. Description of surgical treatment of patients with neurocysticercosis in the brainstem. Following the surgery all previous symptoms resolved. In the definition of neurocysticercosis clinical or surgical treatment is directly related to location, size and number of lesions, thus decreasing mortality and morbidity in this disease.

\section{KEYWORDS}

Neurocysticercosis/etiology, neurocysticercosis/physiopathology, neurocysticercosis/surgery, brain stem.

1 Residentes do Serviço de Neurocirurgia do Hospital São Rafael, Salvador, BA, Brasil.

2 Neurocirurgião do Hospital São Rafael, Salvador, BA, Brasil.

3 Chefe do Serviço de Neurocirurgia do Hospital São Rafael, Salvador, BA, Brasil. 


\section{Introdução}

A cisticercose é considerada uma epidemia mundial. Segundo a Organização Mundial de Saúde (OMS), 50 milhões de indivíduos estão infectados pelo complexo teníase/cisticercose e 50 mil vão a óbito a cada ano. ${ }^{1}$

Cisticercose é a parasitose mais comum que afeta o sistema nervoso central, resultado da infecção humana pelo ovo do parasita Taenia solium. ${ }^{2,3} \mathrm{O}$ cisticerco pode apresentar-se sob duas formas: a cística, contendo escólex no seu interior, conhecido como Cysticercus cellulosae e, em cachos com numerosas vesículas, mas sem o escólex, conhecida como Cysticercus racemosus. ${ }^{1,3,4}$

A neurocisticercose acomete indivíduos de ambos os sexos, em todas as faixas etárias, com predomínio entre 11 e 35 anos. As manifestações clínicas mais importantes são: crises epilépticas (62\%), síndrome de hipertensão intracraniana (38\%), meningite cisticercótica (35\%), distúrbios psiquiátricos (11\%), forma apoplética e endarterítica $(2,8 \%)$ e síndrome medular $(0,5 \%){ }^{1,4}$

As alterações na tomografia de crânio que sugerem neurocisticercose estão na dependência da fase evolutiva da larva. Acredita-se que o cisticerco intraparenquimatoso sofra processo degenerativo em torno de três a seis anos. ${ }^{5,6} \mathrm{~A}$ presença de lesão hipodensa com reforço em anel ou lesão isodensa com reforço homogêneo na fase contrastada é indicativa de degeneração do cisticerco. Após degeneração, ocorre depósito lento e progressivo de cálcio, onde o intervalo médio entre a morte do cisticerco e calcificação radiologicamente perceptível é de 25 meses. A ressonância magnética é mais sensível na detecção de cisticercos cisternais e intraventriculares, com melhor visualização do escólex. ${ }^{5-7}$

$\mathrm{O}$ aprimoramento nos métodos de diagnósticos por imagem e laboratoriais, assim como medicações antiparasitárias, tem proporcionado um melhor plano terapêutico e qualidade de vida desses pacientes.

\section{Caso clínico}

Paciente masculino, 36 anos, natural de Minas Gerais, com quadro de vertigem, alteração do equilíbrio e cefaleia holocraniana há dois anos, com piora progressiva, acompanhado nos últimos três meses do surgimento de hemiparesia esquerda com sinais de liberação piramidal, sem alterações em nervos cranianos. Internado para a investigação etiológica. Ressonância magnética do encéfalo mostrou lesão hipointensa em T1 (Figura 1) e hiperintensa em T2 (Figura 2) envolvendo pedúnculo cerebral e mesencéfalo à direita, sugerindo lesão tumoral cística. Decidido por tratamento cirúrgico devido aos déficits neurológicos em progressão. Realizado acesso subtemporal à direita, com exérese total da lesão, evidenciado em tomografia de crânio (Figura 3), cujo anatomopatológico mostrou tratar-se de neurocisticercose. Decidido pelo não uso de albendazol no pós-operatório pelo risco de reação inflamatória em área eloquente. Paciente obteve evolução satisfatória, com recuperação dos déficits prévios e voltando a realizar suas atividades habituais.

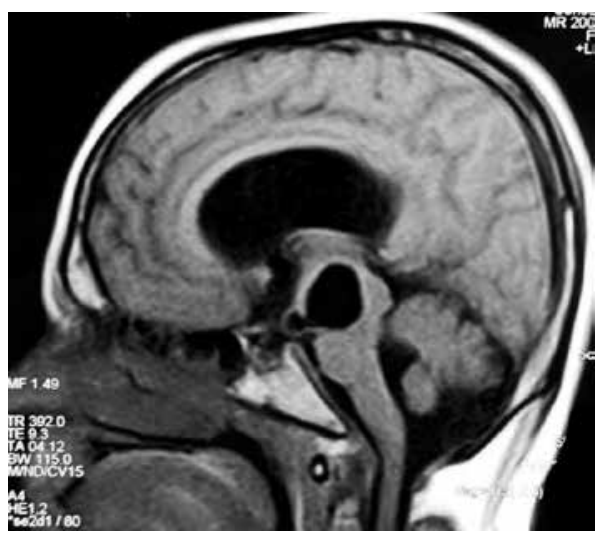

Figura 1 - Ressonância magnética com lesão hipointensa em T1 no tronco cerebral.

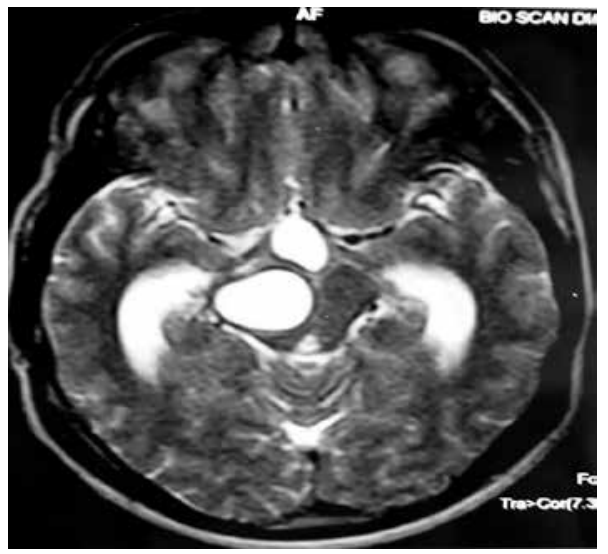

Figura 2 - Ressonância magnética com lesão hiperintensa em T2 no tronco cerebral.

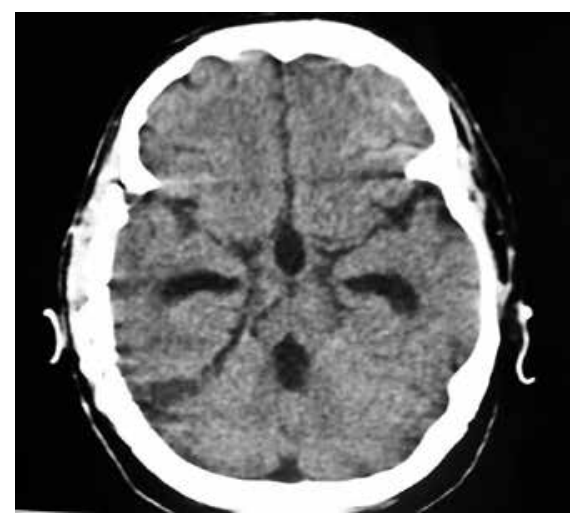

Figura 3 - Tomografia do encéfalo pós-operatória. 


\section{Discussão}

Neurocisticercose é dividida nas formas intra e extraparenquimatosa, em que o tratamento clínico é preferível para lesões císticas em áreas não eloquentes, devido à ocorrência de resposta inflamatória local induzida pela medicação, aumentando o risco de déficit neurológico. ${ }^{1,4,5} \mathrm{O}$ uso de albendazol $(15 \mathrm{mg} / \mathrm{kg} / \mathrm{dia}$ dividido em duas vezes) é mais vantajoso que praziquantel, pois não interage com drogas anticonvulsivantes. Usa-se para lesão única (sete dias), múltiplas (dez a quatorze dias) e subaracnóidea (vinte e oito dias, com resultados controversos) ${ }^{8,9} \mathrm{O}$ antiparasitário é associado a uma terapia antiinflamatória com decadron $(0,1 \mathrm{mg} / \mathrm{kg} / \mathrm{dia})$ ou prednisona $(1 \mathrm{mg} / \mathrm{kg} / \mathrm{dia}){ }^{8}$

Em uma ou mais lesões em áreas não eloquentes, há melhor resultado com terapia medicamentosa (resolução completa em 69\% nas lesões únicas e $41 \%$ das lesões múltiplas), sendo contraindicado o uso de antiparasitários em pacientes com encefalite. Importante afastar lesões intra e extraoculares antes do tratamento.

O tratamento cirúrgico é indicado em paciente com hidrocefalia aguda, em que é necessária derivação ventricular ou remoção do cisto por via endoscópica nos casos menos sintomáticos. ${ }^{10-12}$ Em relação às ressecções abertas, há melhor resposta clínica em lesões com grande efeito de massa, cisto no IV ventrículo e em áreas eloquentes, cujo acesso cirúrgico seja seguro, pois a resposta inflamatória do tratamento medicamentoso traria déficits neurológicos por lesão dessas áreas. ${ }^{13}$

\section{Conclusão}

A neurocisticercose é rara no tronco cerebral, onde seu tratamento encontra-se intimamente ligado à sua localização e ao número de lesões. Deve ser enfatizada a importância na escolha do método de tratamento, devendo-se incluir no diagnóstico diferencial de lesões neoplásicas.

\section{Conflito de interesses}

Os autores declaram inexistência de conflito de interesses na realização deste trabalho.

\section{Referências}

1. Colli BO, Carlotti Junior CG. Fisiopatologia, diagnóstico e tratamento da cisticercose do sistema nervoso central. Temas Atuais Neurocirurgia. 2003;1:4-28.

2. Colli BO, Carlotti CG Jr, Assirati JA Jr, Machado HR, Valença M, Amato MC. Surgical treatment of cerebral cysticercosis: long-term results and prognostic factors. Neurosurg Focus. 2002;12(6):e3.

3. Medina TM, Degiorgio CM. Introduction to neurocysticercosis: a worldwide epidemic. Neurosurg Focus. 2002,12:e1.

4. Garcia HH, Pretell EJ, Gilman RH, Martinez SM, Moulton $\mathrm{LH}$, Del Brutto OH, et al. A trial of antiparasitic treatment to reduce the rate of seizures due to cerebral cysticercosis. $\mathrm{N}$ Engl J Med. 2004;350(3):249-58.

5. Minguetti G, Ferreira MV. Computed tomography in neurocysticercosis. J Neurol Neurosurg Psychiatry. 1983;46(10):936-42.

6. Rodriguez-Carbajal J. Palacios E, Zee C. Neuroradiology of cysticercosis of the central nervous system. In: Palacios E, Rodriguez-Carbajal J, Taveras JM, editors. Cysticercosis of the central nervous system. Springfield: Charles C. Thomas; 1983. p. $101-43$.

7. Nash TE, Pretell EJ, Lescano AG, Bustos JA, Gilman RH, Gonzalez AE, et al. Perilesional brain oedema and seizure activity in patients with calcified neurocysticercosis: a prospective cohort and nested case-control study. Lancet Neurol. 2008;7(12):1099-105.

8. Carpio A, Kelvin EA, Bagiella E, Leslie D, Leon P, Andrews $\mathrm{H}$, et al. Effects of albendazole treatment on neurocysticercosis: a randomised controlled trial. J Neurol Neurosurg Psychiatry. 2008;79(9):1050-5.

9. Recommendations of the International Task Force for Disease Eradication. MMWR Recomm Rep. 1993;42(RR16):1-38.

10. Rangel-Castilla L, Serpa JA, Gopinath SP, Graviss EA, Diaz-Marchan P, White AC Jr. Contemporary neurosurgical approaches to neurocysticercosis. Am J Trop Med Hyg. 2009;80(3):373-8.

11. Kelley R, Duong DH, Locke GE. Characteristics of ventricular shunt malfunctions among patients with neurocysticercosis. Neurosurgery. 2002;50(4):757-61.

12. Del Brutto OH, Roos KL, Coffey CS, García HH. Metaanalysis: cysticidal drugs for neurocysticercosis: albendazole and praziquantel. Ann Intern Med. 2006;145(1):43-51.

13. Verma A, Misra S. Outcome of short-term antiepileptic treatment in patient with solitary cerebral cysticercus granuloma. Acta Neurol Scand. 2006;113(3):174-7.

Endereço para correspondência

Luiz Eduardo Ribeiro Wanderley Filho

Av. Bosque Imperial, 313, bloco 11, ap. 203

41250-480 - Salvador, BA, Brasil

E-mail: luizeduardowand@yahoo.com.br 